\title{
Different selective pressures lead to different genomic outcomes as newly-formed hybrid yeasts evolve
}

\author{
Jeff S Piotrowski ${ }^{1,3}$, Saisubramanian Nagarajan ${ }^{2,3}$, Evgueny Kroll ${ }^{3}$, Alison Stanbery ${ }^{3}$, Kami E Chiotti ${ }^{3}$, \\ Arthur L Kruckeberg ${ }^{4}$, Barbara Dunn ${ }^{5}$, Gavin Sherlock ${ }^{5}$ and Frank Rosenzweig ${ }^{3^{*}}$
}

\begin{abstract}
Background: Interspecific hybridization occurs in every eukaryotic kingdom. While hybrid progeny are frequently at a selective disadvantage, in some instances their increased genome size and complexity may result in greater stress resistance than their ancestors, which can be adaptively advantageous at the edges of their ancestors' ranges. While this phenomenon has been repeatedly documented in the field, the response of hybrid populations to long-term selection has not often been explored in the lab. To fill this knowledge gap we crossed the two most distantly related members of the Saccharomyces sensu stricto group, S. cerevisiae and S. uvarum, and established a mixed population of homoploid and aneuploid hybrids to study how different types of selection impact hybrid genome structure.
\end{abstract}

Results: As temperature was raised incrementally from $31^{\circ} \mathrm{C}$ to $46.5^{\circ} \mathrm{C}$ over 500 generations of continuous culture, selection favored loss of the $S$. uvarum genome, although the kinetics of genome loss differed among independent replicates. Temperature-selected isolates exhibited greater inherent and induced thermal tolerance than parental species and founding hybrids, and also exhibited ethanol resistance. In contrast, as exogenous ethanol was increased from $0 \%$ to $14 \%$ over 500 generations of continuous culture, selection favored euploid S. cerevisiae $\times$ S. uvarum hybrids. Ethanol-selected isolates were more ethanol tolerant than S. uvarum and one of the founding hybrids, but did not exhibit resistance to temperature stress. Relative to parental and founding hybrids, temperature-selected strains showed heritable differences in cell wall structure in the forms of increased resistance to zymolyase digestion and Micafungin, which targets cell wall biosynthesis.

Conclusions: This is the first study to show experimentally that the genomic fate of newly-formed interspecific hybrids depends on the type of selection they encounter during the course of evolution, underscoring the importance of the ecological theatre in determining the outcome of the evolutionary play.

\section{Background}

Interspecific hybridization occurs in every eukaryotic kingdom and can lead to reticulated rather than branching phylogenies [1,2]. Hybrid progeny are often at a strong selective disadvantage (e.g., they may be sterile or have reduced viability). However, in some instances the increased genome size and complexity of interspecific hybrids may result in greater fecundity and/or adaptive flexibility than either ancestral species [3], particularly at

\footnotetext{
* Correspondence: Frank.Rosenzweig@mso.umt.edu

${ }^{3}$ Division of Biological Sciences, The University of Montana, Missoula MT 59812, USA

Full list of author information is available at the end of the article
}

the edges of the ancestral species' range, where they are more likely to encounter stress [4]. This phenomenon is amply documented in the agricultural literature as well as in field-based evolutionary studies [1,2,5-10]. Laboratory studies of interspecific hybridization have largely been confined to the Drosophila species complex, where foundational studies have shaped our understanding of the genetic basis for pre-zygotic and post-zygotic reproductive isolation [11-13]. Long-term experimental studies aimed at discerning the evolutionary trajectories open to newly-formed hybrids under different types of selection are lacking, a knowledge gap due in part to the scarcity of hybrid eukaryotes that have the short generation time
C Biomed Central

() 2012 Piotrowski et al; licensee BioMed Central Ltd. This is an Open Access article distributed under the terms of the Creative Commons Attribution License (http://creativecommons.org/licenses/by/2.0), which permits unrestricted use, distribution, and reproduction in any medium, provided the original work is properly cited. 
and ease of preservation needed to undertake experiments lasting hundreds of generations.

Experimental evolution studies using microbes have enlarged our understanding of the tempo of adaptive change [14-16], the shape of the adaptive landscape [17], and the manner in which genotypes navigate that landscape [18]. Increasingly, the budding yeast Saccharomyces cerevisiae has become a favored model for such studies because of its genetic tractability, arsenal of post-genomic tools, and homology of many of its genes to those in higher eukaryotes. Yeast's short generation time, simple, heterogonic life cycle and ease of preservation ideally suit it for studying evolution in the laboratory [16,19-21], where it has yielded insights into the physiology of adaptive traits [22] and how genome structure evolves under selection [23,24].

The 7 members of the Saccharomyces sensu stricto group, though closely related, have long been recognized as biological species by virtue of their post-zygotic reproductive isolation $[25,26]$. While bona fide representatives of each species are easily recovered from nature [27], retrospective comparative genomics studies $[28,29]$ suggest that interspecific hybridization has occurred repeatedly during the group's evolutionary history. Indeed, the lager yeast $S$. pastorianus, which likely arose $\sim 500-600$ years ago [30], is a natural hybrid of two species, S. cerevisiae and the newly discovered S. eubayanus [26]. Saccharomyces hybrids have most often been studied with the aim of developing industrially useful traits [31,32], typically by focusing on the physiology of single clones. However, Grieg et al. (2002) showed that the sensu stricto group could also be used to investigate hybrid speciation in the lab [33]. Currently lacking, however, are prospective studies of interspecific hybrid evolution under different types of selection, using either single clones or, more realistically, populations of hybrid clones, such as one might expect to arise in natural hybrid zones.

The two most distantly-related members of the Saccharomyces sensu stricto group, S. cerevisiae and S. uvarum (formerly S. bayanus var. uvarum [26]), are largely syntenic, exhibit $80 \%$ and $62 \%$ nucleotide identity in coding regions and intergenic regions, respectively, and are thought to have diverged $\sim 20$ million years ago [34]. The two species differ in their stress tolerances, with $S$. cerevisiae being much more thermal tolerant [35-37] and slightly more ethanol tolerant [38]; S. cerevisiae $\mathrm{x}$ $S$. uvarum hybrids can exhibit greater ethanol tolerance than either parental species [39]. These genetic and phenotypic differences, coupled with the availability of genomic resources for both species, make $S$. cerevisiae $\mathrm{x}$ S. uvarum hybrids an attractive system in which to investigate how newly-formed hybrid genomes evolve under different types of stress. We therefore sporulated a S. cerevisiae $\mathrm{x}$ S. uvarum hybrid and mass-mated the progeny to create a pool of S. cerevisiae x S. uvarum homoploid and aneuploid hybrids that was used to found six replicate populations. Because fungi in nature are chronically nitrogen limited $[40,41]$, experimental populations were evolved in a glucose-sufficient, nitrogen-limited 'common garden;' three were subjected to incremental increases in temperature, and three were subjected to incremental increases in ambient ethanol. Because the two ancestral species and their F1 hybrid exhibit differential sensitivity to temperature and ethanol, and because stress has been shown to increase mitiotic recombination, including chromosome missegregation [42], we hypothesized that the two selective pressures would lead to different genomic outcomes. As prior experiments in S. cerevisiae had shown that thermal tolerance confers cross-protection against other types of stress [37,43], we further hypothesized that hybrids evolving under temperature selection would not only become more thermal tolerant than their ancestors but also exhibit greater ethanol tolerance, and vice versa.

We tested these hypotheses using an integrated approach that combined physiological assays with analysis of genome structure by Clamped Homogeneous Electric Field (CHEF) electrophoresis and array-Comparative Genomic Hybridization (a-CGH). Consistent with our primary hypothesis, temperature selection resulted in loss of the $S$. uvarum genome from interspecific hybrids, while ethanol selection resulted in yeast that retained essentially both $S$. cerevisiae and $S$. uvarum genomes. Consistent with our secondary hypothesis, cross-protection to ethanol was evident in the temperature selected isolates; however, cross-protection to thermal stress was not observed among ethanol-selected clones. Thus, as hybrid populations evolve under different selection pressures their fate may depend not only on the outcome of competition between individual variants, but also on the outcome of competition between the ancestral genomes themselves.

\section{Results and Discussion \\ Creation of the founder hybrid population and experimental design}

An overview of the experimental design is presented in Figure 1. A pool of approximately 10,000 interspecific hybrid clones was created using two prototrophic parental strains: CEN.PK (Saccharomyces cerevisiae) [44] and CBS7001 (S. uvarum) [30]. We crossed haploid derivatives of the parental strains using double antibiotic selection and verified by pulsed-field gel and by a-CGH that the resulting F1 hybrid contained a complete genome from each parent (data not shown). The hybrid population was then obtained by sporulating the F1 hybrid and removing vegetative F1 hybrid cells as described in Methods and in [33]. F1 hybrids sporulate at low efficiency, thus sporulation was done en masse. Because spore germination occurred in contact with other spores (see Methods), it is 


\section{S. cerevisiae CEN.PK YEp352-KanMX G418 + Hygromycin selection $\downarrow$ \\ S. uvarum \\ CBS7001 \\ YEp352-hph}

F1

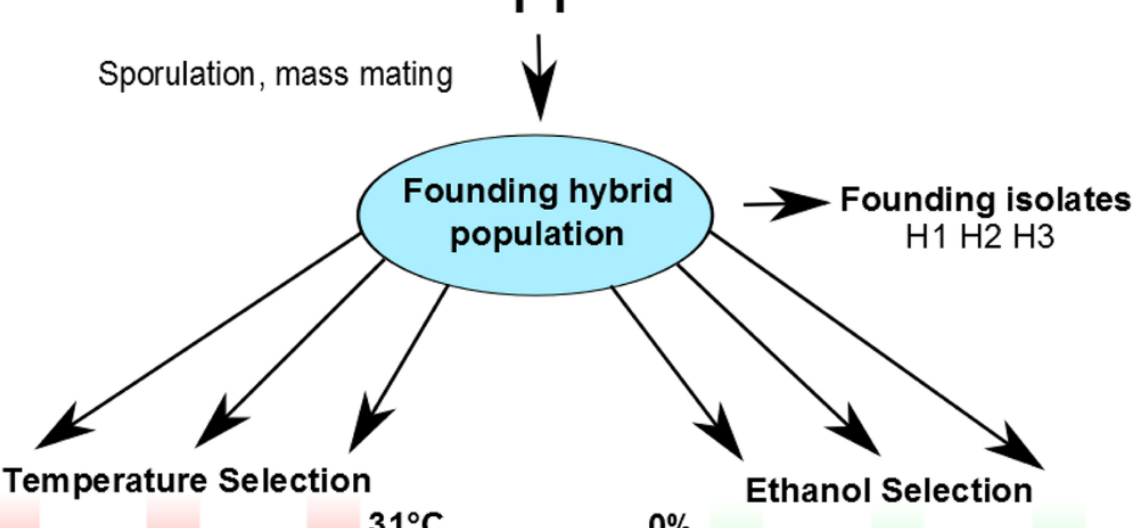

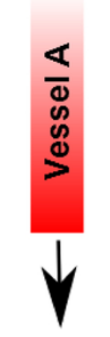

A1 A2 A3

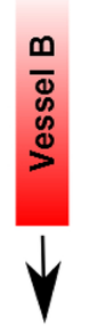

B1 B2 B3

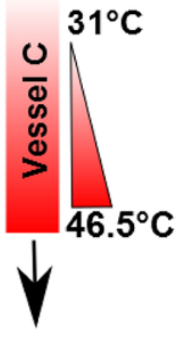

$\mathrm{C} 1 \mathrm{C} 2 \mathrm{C} 3$

\section{0 generation isolates}

Figure 1 Experimental design: Response of a genetically heterogeneous hybrid population to temperature or ethanol selection. $S$. cerevisiae (CEN.PK) and S. uvarum (CBS7001) were transformed with plasmids conferring G418 and hygromycin resistance, respectively. The two species were mated, then placed under dual antibiotic selection to screen for a viable F1 interspecific hybrid. This F1 was sporulated and the spores allowed to diploidize by mass mating. The resulting genetically mixed hybrid population was used to found 3 experimental populations for temperature selection and 3 experimental populations for ethanol selection. After 500 generations of incremental increases in either temperature or ethanol, three clones were isolated from each experimental vessel and their stress tolerance compared to that of the two ancestral species, an F1 interspecific S. cerevisiae/S. uvarum hybrid, and three isolates from the founding hybrid population.

probable that most of the viable and mating-proficient gametes mated, resulting in a genetically mixed hybrid population that was largely diploid. Nevertheless, we cannot rule out the possibility that this population may have contained a low proportion of unmated F2 gametes. Additionally, despite our efforts to obtain a pure F2 population, some F1 cells did survive the procedure.

Hybrid cells with complete or almost-complete chromosomal complements tended to grow faster than those cells that were multiply aneuploid (data not shown). Consequently, our starting inoculum was a complex mixture of F1 and F2 hybrids having different levels of aneuploidy and specific growth rates. A mixture of F1, haploid and aneuploid F2 hybrids is an evolutionarily relevant starting point for our experiments, inasmuch as it mimics the genetic complexity one might expect to see within a hybrid zone [45]. We refer to this pool as the founder hybrid population. For purposes of comparison with clones subsequently isolated after many generations of selective growth, three isolates were randomly chosen from the founder hybrid population and denoted $\mathrm{H}_{1}, \mathrm{H}_{2}$, and $\mathrm{H}_{3}$. All strains used in this study are listed in Additional file 1: Table S1.

Steady-state population size declines as evolving hybrid populations respond to increases in either temperature or ethanol

In the wild, fungi including yeast are believed to live in a state of chronic nitrogen limitation [40], as do yeast in large-scale industrial fermentations $[46,47]$. To mimic 
these conditions we performed our selection experiments in a 'common garden' in which nitrogen in the form of ammonia was limiting. Cell density in evolving glucose-sufficient, $\mathrm{NH}_{4}$-limited populations declined as either temperature or ethanol was increased over the course of 500 generations (Figure 2). At the onset of experiments, cells cultured at $31^{\circ} \mathrm{C}$ attained steady state cell densities of $1.50 \times 10^{8} \pm 1.1 \times 10^{7}$ cells ml $^{-1}(\mathrm{~N}=6$, Mean \pm S.E.). Following incremental temperature selection up to $46.5^{\circ} \mathrm{C}$ (Figure 2A) or ethanol amendment up to $14 \%$ (Figure $2 \mathrm{~B}$ ), steady state cell densities fell to 2.0 $\times 10^{5} \pm 1.0 \times 10^{5}$ cells ml $^{-1}$ and $1.87 \times 10^{5} \pm 1.43 \times 10^{5}$ cells $\mathrm{ml}^{-1}$ respectively, a decrease of 3 orders of magnitude. Indeed, at these low densities it was necessary to decrease chemostat dilution rate in order to prevent yeast populations from washing out of culture vessels, an indication of diminished growth rate under stress. We observed no dramatic between-vessel differences in population parameters, suggesting a similar response to ethanol and temperature selection in independent experiments founded with the same genetically heterogenous inoculum.

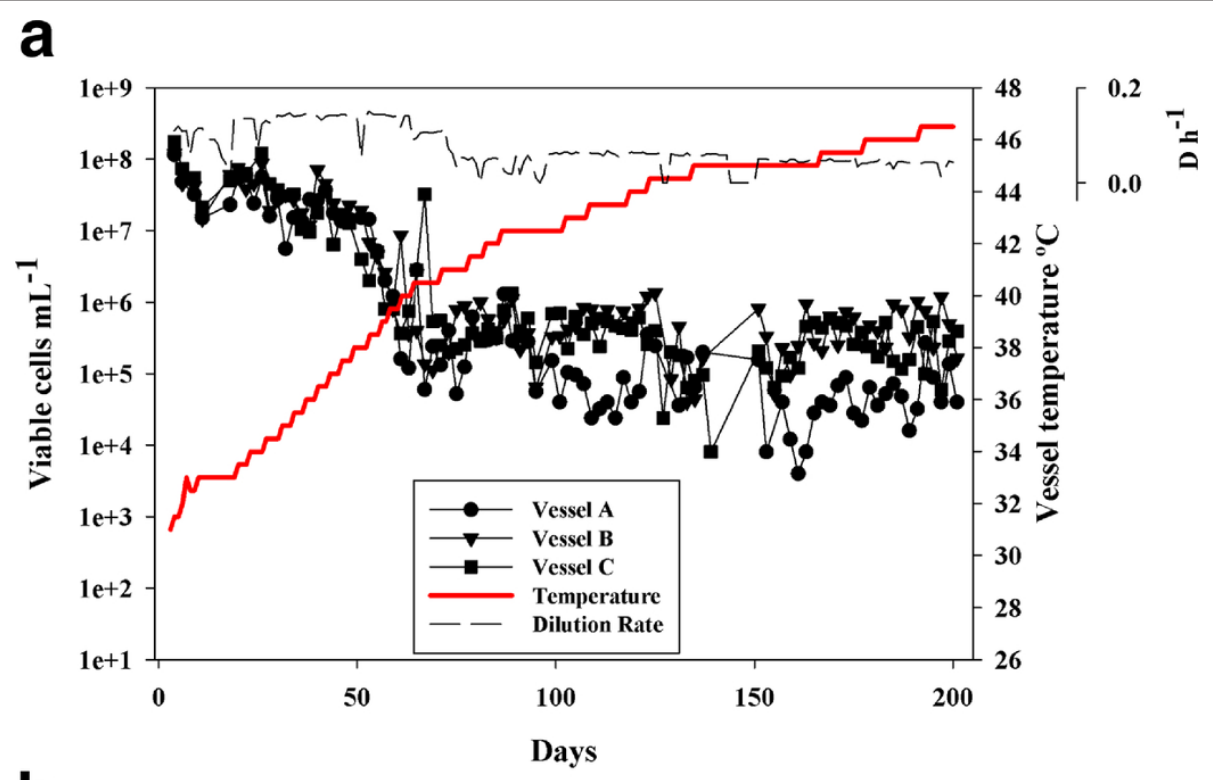

b

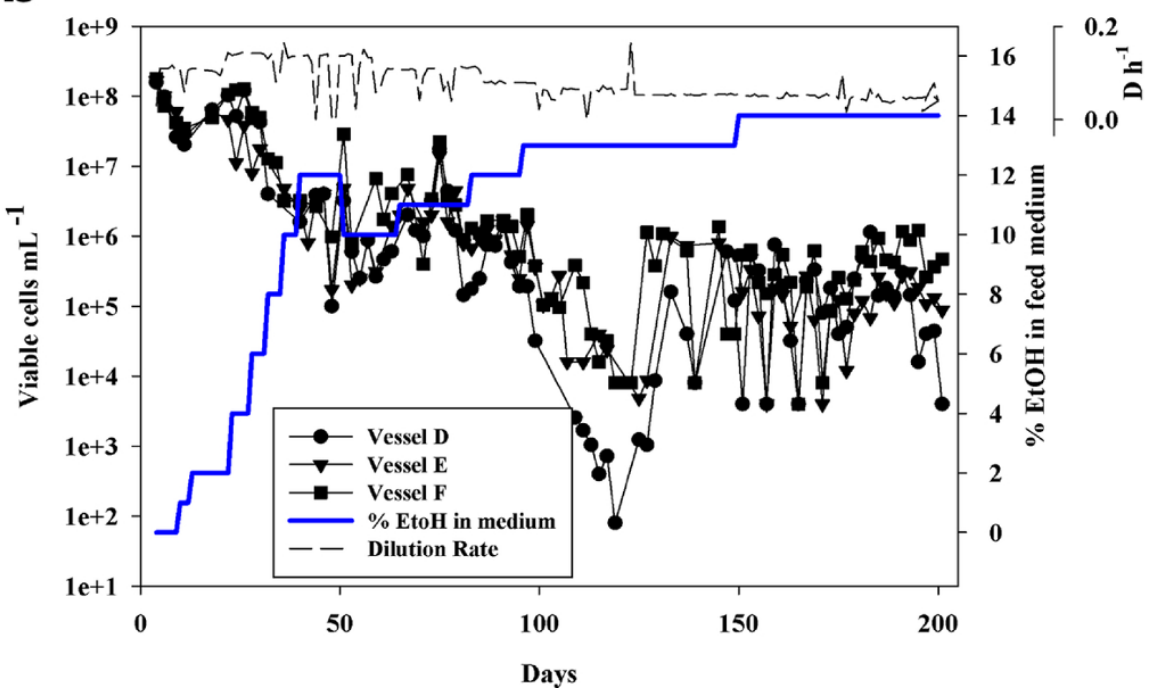

Figure 2 Experimental evolution of S. cerevisiae X S. uvarum hybrids under increasing temperature (A) and ethanol (B). Six replicate populations, founded by the same genetically diverse hybrid pool (see Figure 1), were selected for 200 days ( 500 generations) under glucosesufficient, nitrogen-limiting conditions. Population size was estimated as viable cells $\mathrm{mL}^{-1}$. The dashed line represents dilution rate, $D$, in $\mathrm{h}^{-1}$; the solid red line represents vessel temperature (A), the solid blue line represents ethanol content of the medium fed to evolving populations (B) (Mean \pm S.E.). 
Yeasts recovered from temperature selection are more thermotolerant than members of the founder population To assess for heritable adaptations to thermal stress, we compared growth of 3 representatives of the founder hybrid population to that of 9 isolates selected at elevated temperature (3 single colony isolates from each experimental population, see Methods and Figure 1, Additional file 1: Table S1). When cultured at $40^{\circ} \mathrm{C}$ to stationary phase $(48 \mathrm{~h})$ in liquid low-nitrogen, minimal medium (Figure 3), temperature-selected isolates consistently showed greater thermal tolerance than $S$. uvarum, the F1 hybrid and members of the founder population. As a group, evolved isolates exhibited significantly greater yield than unevolved hybrids $(P=0.03, T$-Test $)$. Isolates from vessel $C$ had significantly higher cell yield at $40^{\circ} \mathrm{C}$ than all other isolates including the $S$. cerevisiae parent $(P<0.05$, ANOVA followed by Tukey's HSD).

Yeast acquire thermal tolerance following entry into stationary phase [48], as well as following exposure to low levels of heat or other types of stress [49,50]. Natural and laboratory variants can also exhibit strain-specific differences in thermal tolerance owing to different genetic backgrounds [35]. To test whether selection had resulted in yeast that were inherently more thermal tolerant than members of the founder population we evaluated survivorship of cultures exposed to $48^{\circ} \mathrm{C}$ for 5 $\mathrm{h}$ (Figure 4A). Following 1 and $2 \mathrm{~h}$ incubation at $48^{\circ} \mathrm{C}$, all temperature selected isolates exhibited significantly greater survivorship than their ancestral strains $(P<0.05$, Tukey's HSD). As no $S$. uvarum cells survived even $1 \mathrm{~h}$ at $48^{\circ} \mathrm{C}$, they are not represented on this graph. We also wished to test the hypothesis that temperature-selected isolates would exhibit greater phenotypic plasticity under thermal stress. To do this, we induced thermal tolerance by first exposing cells to a sub-lethal elevated temperature $\left(37^{\circ} \mathrm{C}\right)$ for $1 \mathrm{~h}$ prior to exposure to a $48^{\circ} \mathrm{C}$ heat shock (Figure 4B). Aside from S. uvarum, which again had no surviving cells, all other strains exhibited thermotolerance. Temperature-selected clones from populations A and $\mathrm{C}$ displayed higher survivorship than the S. cerevisiae parent and representative hybrids from the founding hybrid population at $2 \mathrm{~h}(P<0.05$, Tukey's HSD $)$; clones from vessel $B$ had significantly greater survivorship than founding hybrids $(P<0.05$, Tukey's HSD). Thus, selection on a population of interspecific hybrids resulted in yeast that not only sustain growth at higher temperature than members of the founder population, but also are intrinsically more heat-shock resistant and able to acquire greater heat-shock resistance physiologically by induction.

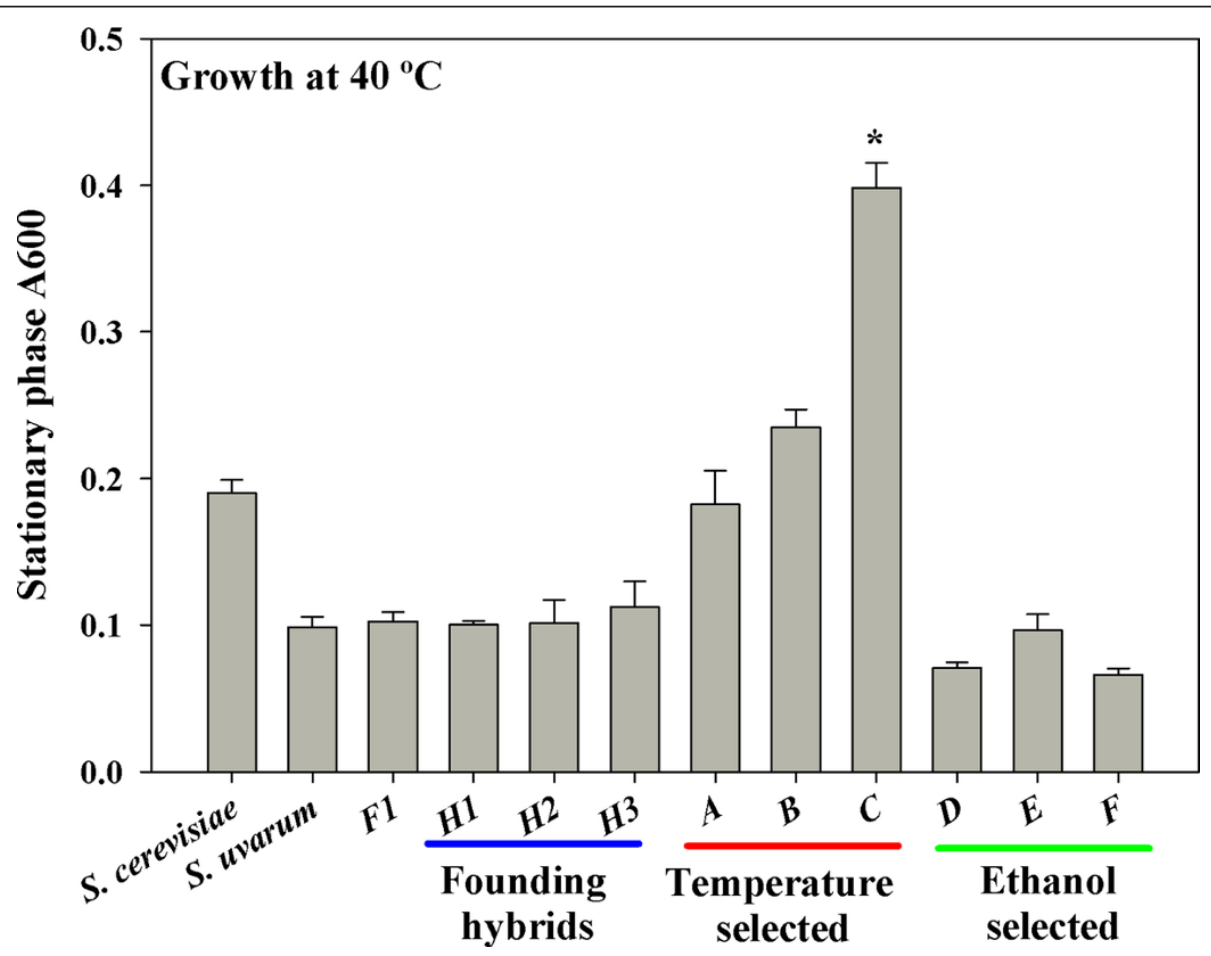

Figure 3 Growth of evolved isolates at elevated temperature. Culture density $\left(A_{600}\right)$ of parental, $F 1$, founding and selected hybrid strains from each experimental population following $48 \mathrm{~h}$ growth in liquid, low-nitrogen, minimal medium at $40^{\circ} \mathrm{C}$. Asterisk indicates significantly different growth, relative to all other isolates $(P<0.05$, Mean \pm S.E.). 

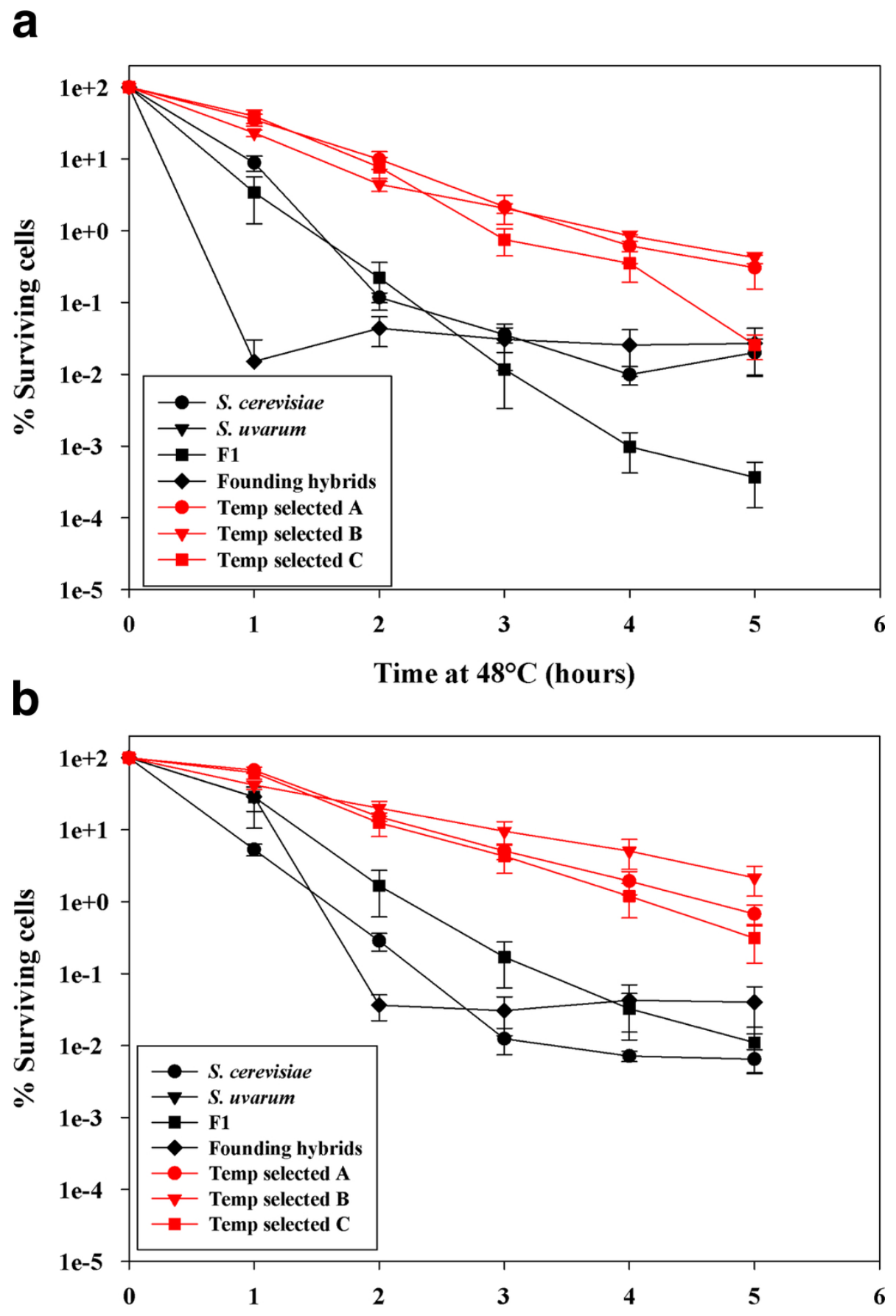

Time at $48^{\circ} \mathrm{C}$ following $1 \mathrm{hr}$ pre-treatment at $37^{\circ} \mathrm{C}$ (hours)

Figure 4 Viability of parental species, F1 and founder interspecifc hybrids, and temperature-selected isolates in liquid culture following exposure to $48^{\circ} \mathrm{C}$. (A) Inherent thermal tolerance. A sample of each culture was diluted and plated on $2 \%$ YPD every hour for $5 \mathrm{~h}$. No viable S. uvarum cells were detected at the $1 \mathrm{~h}$ time-point, thus S. uvarum data are not presented. After $2 \mathrm{~h}$ survivorship of the selected isolates was greater than all other isolates $(P<0.05)$ (B) Induced thermal tolerance. Following overnight culture at $25^{\circ} \mathrm{C}$, cells were incubated for $1 \mathrm{~h}$ at $37^{\circ} \mathrm{C}$ prior to exposure to $48^{\circ} \mathrm{C}$. Samples of each culture were then diluted and plated every hour for 5 h. No viable $\mathrm{S}$. uvarum cells were detected at the $1 \mathrm{~h}$ time-point, thus S. uvarum data and are not presented. After $2 \mathrm{~h}$, survivorship of the selected isolates was greater than the founding hybrids $(P<0.05)$. Red lines and filled symbols represent the temperature-selected isolates. Experiments were performed in triplicate (Mean \pm S.E.). 
Yeasts recovered from ethanol selection do not appear to be more ethanol tolerant than members of the founder population

The manner and extent to which interspecific hybrid populations responded to ethanol selection was less clear-cut. When cultured until stationary phase (48 h) in liquid, low-nitrogen minimal medium amended with $8 \%$ ethanol, culture densities among ethanol-selected isolates were statistically indistinguishable from those of $S$. cerevisiae, the F1 interspecific hybrid, founder population isolates $\mathrm{H} 1$ and $\mathrm{H} 2$, and the temperature-selected isolates (Additional file 2: Figure S1). Only the $S$. uvarum parent and founder isolate $\mathrm{H} 3$ proved to be ethanol sensitive by this assay $(P<0.05$, Tukey's HSD). Furthermore, temperature selected isolates were no less ethanol tolerant than ethanol-selected isolates.

\section{Temperature and ethanol selection can lead to changes in cell wall integrity}

Heat-shock studies in S. cerevisiae have shown that yeast in prolonged stationary phase become resistant to heat and other stresses owing to progressive changes in cell wall structure that alter its mannoprotein content, increase the amount of chitin incorporated and augment the $\beta-1,6$ glucan fraction [51,52]. Phenotypically, such cell wall changes manifest as increased resistance to zymolyase digestion [51]. We therefore evaluated resistance of ancestral and evolved yeast to zymolyase digestion using the spheroplast assay described by [53]. Over the course of a $1 \mathrm{~h}$ incubation, the cell walls of temperature-selected isolates from vessels $\mathrm{A}, \mathrm{B}$, and $\mathrm{C}$ consistently exhibited higher average resistance to enzymatic dissolution than did either parental strain, the F1, or isolates from the founder hybrid population (Figure 5A). As a group, temperature-selected strains had significantly higher remaining absorbance percentage $(48 \pm 6 \%)$ after $1 \mathrm{~h}$ than founder population hybrids $(20 \pm 4 \%),(P<0.05$, T-Test $)$.

We also observed that temperature-selected isolates were more resistant to Micafungin, a drug that targets $\beta-1,3$ glucan synthesis [54] (Additional file 3: Figure S2). Isolates from vessels $\mathrm{A}$ and $\mathrm{C}$ exhibited significantly greater resistance to this drug than $S$. cerevisiae, $S$. uvarum, the $\mathrm{F} 1$ and founding hybrids $(P<0.05$, Tukey's HSD). This suggests that changes in cell wall composition or deposition contribute to the thermal tolerant phenotype observed in isolates from vessels $\mathrm{A}$ and $\mathrm{C}$, whereas isolates from vessel $B$ may have evolved a different resistance mechanism. This suggestion is supported by the fact that vessel B isolates, while not Micafungin-resistant, did exhibit resistance to zymolyase digestion.

Although interspecific hybrid populations responded weakly, if at all, to ethanol selection, we nevertheless assayed for heritable changes in cell wall integrity. Interestingly, similar to members of the temperature-selected populations, ethanol-selected isolates were more zymolyase resistance than parental species, the F1 hybrid and founder isolates (Figure 5B), though only isolates from vessel E showed significantly greater zymolyase resistance than founding hybrids after $1 \mathrm{~h}$ of treatment $(P<$ 0.05, T-test). Strains isolated from ethanol-selected populations showed no difference in Micafungin sensitivity relative to the parental species, the $S$. cerevisiae $\mathrm{x}$ S. uvarum F1 hybrid, or isolates drawn from the founder population (Figure 5B).

\section{Temperature and ethanol selection lead to different genomic outcomes}

We initially assessed genome-wide changes in evolving hybrid populations by analyzing their electrokaryotypes using Contour-clamped Homogeneous Electric Field (CHEF) electrophoresis. CHEF analysis indicated that the founding hybrid population consisted of a mixture of $\mathrm{F} 1$ and F2 spore progeny (Figure 6A). Following 500 generations of temperature selection under nitrogen limitation (Figure 6B), populations in vessels A and B appeared to be karyotypically monomorphic, whereas the population in vessel $C$ was polymorphic (e.g., Figure 6B, lane 20), albeit with a numerically dominant clone. Interestingly, the numerically dominant karyotype in each population (e.g., Figure 6B, lane 7) could be readily distinguished from the dominant clone in each of the other two populations (e.g., Figure 6B, lanes 8 and 17), indicating that while cell growth phenotypes might have been similar among vessels, genome content was not. In other words, different genomic architectures can solve the problem of growing at elevated temperature under nitrogen limitation. Further inspection of the karyotypes suggested that clones from populations in vessels $A$ and $C$ had lost virtually all the $S$. uvarum chromosomal complement of their genomes, keeping only the $S$. cerevisiae genome, while isolates from vessel B retained not only the entire $S$. cerevisiae genome, but also an additional chromosome (Figure 6B, lane 8). The dramatic large-scale genomic changes observed during temperature selection contrast sharply with ethanol selection, where after 500 generations, hybrids showed few rearrangements and no apparent loss of either parental genome (Figure 6C).

\section{Temperature selection on the founding hybrid population favors loss of the S. Uvarum genome}

Because changes in cell size and budding pattern (data not shown) suggested that temperature-adapted interspecific hybrids had become haploid, genome content was assessed by flow cytometry of SYTOX green-stained cells. As anticipated, the parental species, the F1 interspecific hybrid, and a representative from the founder hybrid population were all diploid, whereas temperatureselected clone A1 was haploid (Figure 7). To estimate the 

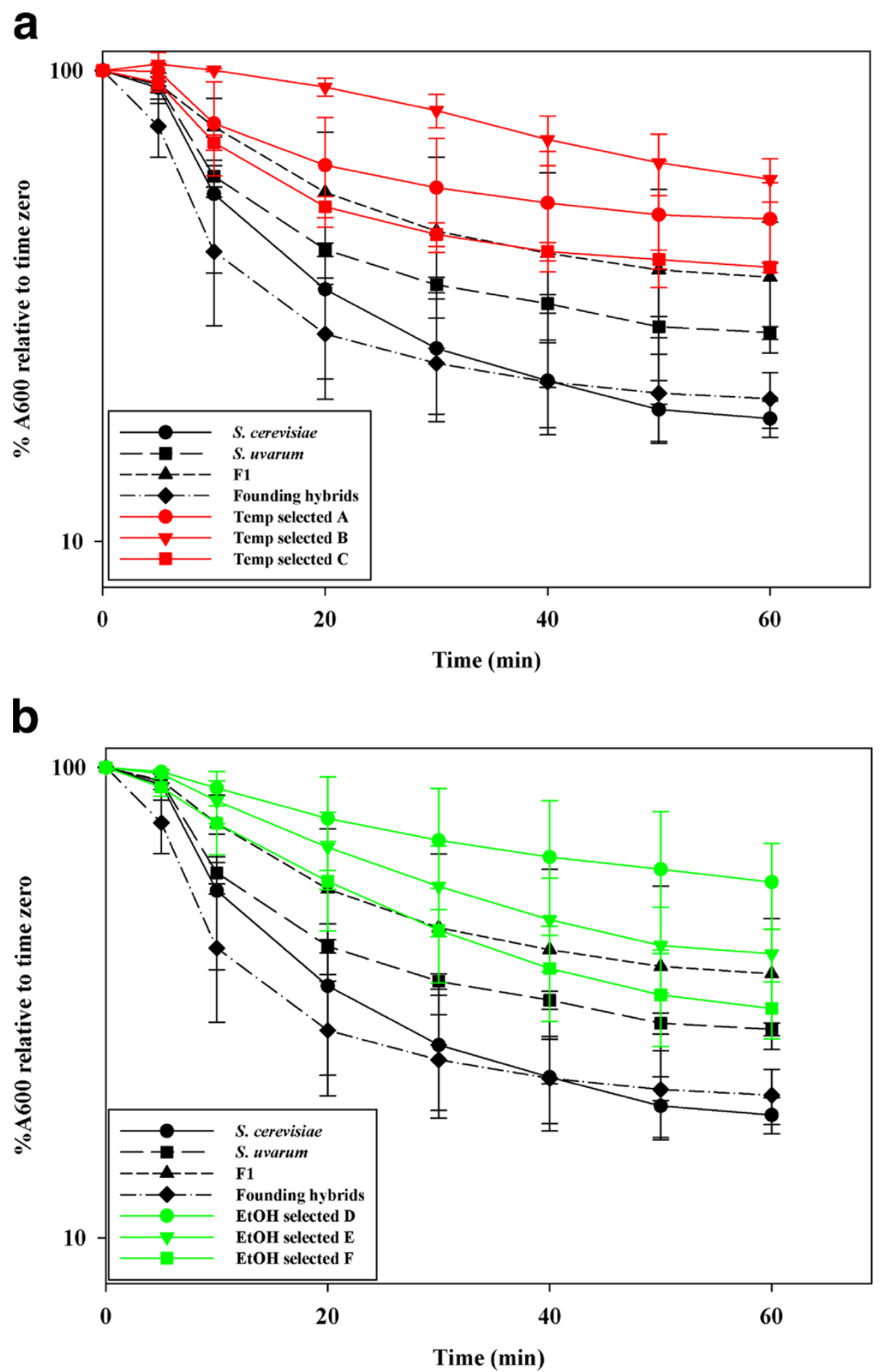

Figure 5 Resistance of parental, founding hybrid, and (A) temperature-selected or (B) ethanol-selected hybrid yeast to zymolyase. Cell wall dissolution was assayed by exposing yeast to zymolyase, placing them in spheroplast buffer at $37^{\circ} \mathrm{C}$, then monitoring decrease in absorbance over time at $\lambda=600 \mathrm{~nm}$, relative to $T_{0}$. Each point is the mean $(n=3)$ of isolates from each selection vessel ( 1 from each vessel), 3 founding isolates, the F1, or parental isolates (Mean \pm S.E.).

approximate number of generations required for haploid cells to become prevalent in experimental populations we performed flow cytometry on archived population samples (rather than on single clones). We found that although populations in vessels A, B and C were founded with the same inoculum, the transition from diploidy to 

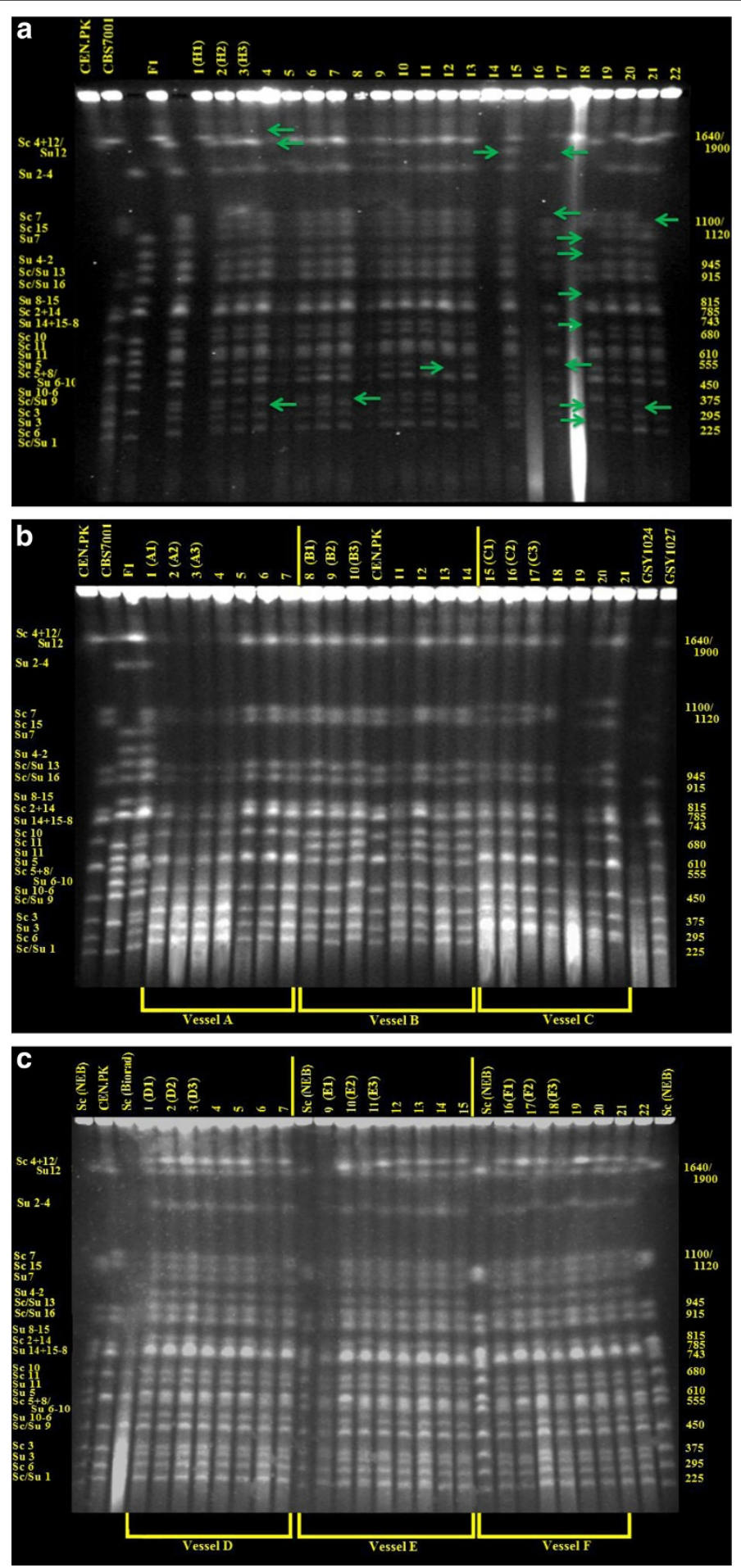

Figure 6 (A) CHEF karyotypes of the founder population, $25^{\circ} \mathrm{C}$. At left are the karyotypes of parental strains, S. cerevisiae CEN.PK, S. uvarum CBS7001, and their F1 interspecific hybrid. In lanes 3-24 are a set of random clones isolated from the common interspecific hybrid pool used to found all replicate populations. Green arrows indicate instances of karyotypic diversity (B) CHEF karyotypes after $\mathbf{5 0 0}$ generations of nitrogen limited, glucose sufficient culture with increasing temperature. 7 random clones were isolated from each experimental population. (C) CHEF karyotypes after $\mathbf{5 0 0}$ generations of nitrogen limited, glucose sufficient culture with increasing ethanol. 7 random clones were isolated from each experimental population. Three different $S$. cerevisiae reference markers are included: the parent CEN.PK, one from New England Biosystems (SC NEB), and from Biorad (SC Biorad). 


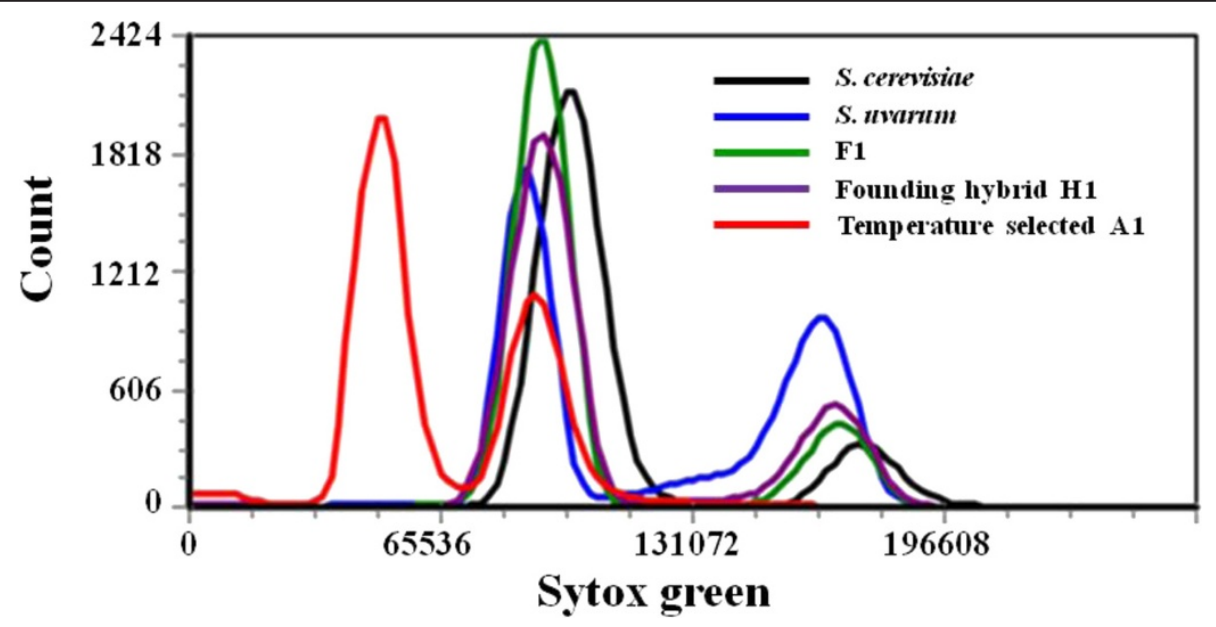

Figure 7 Ploidy of temperature-selected isolates differs from that of parental species, the F1 interspecific hybrid, and a founder hybrid. A representative isolate from Vessel $\mathrm{A}$ is presented. The transition from diploidy to haploidy in each temperature-selected population is presented in Figure S3. Cells were stained with SYTOX and sorted by flow cytometry.

haploidy occurred at a different time in each (Additional file 4: Figure S3). The last point at which population A was predominantly diploid was 101 generations $\left(37.5^{\circ} \mathrm{C}\right)$, population $\mathrm{B}, 115$ generations $\left(37^{\circ} \mathrm{C}\right)$ and population $\mathrm{C}$, 43 generations $\left(33^{\circ} \mathrm{C}\right)$. Moreover, the number of additional generations subsequently required for haploid, or nearly haploid genomes, to become prevalent differed among replicate experiments. An additional 123 generations ensued before population A became mostly haploid, whereas this occurred in population B within 20 generations. And although haploids arose earliest in population C, 52 additional generations elapsed before the population became mostly haploid. Because the kinetics of transition between diploidy and haploidy differed among populations, fitness relationships between haploids and diploids co-evolving in each vessel likely came to differ, even though all were founded with the same inoculum and maintained under identical conditions.

We speculate that haploids arose when diploid hybrids sporulated under aerobic nitrogen limitation. Alternatively, a rare haploid, or nearly-haploid, F1 gamete may have been present in the initial founder hybrid population. If such a gamete contained only $S$. cerevisiae chromosomes and no $S$. uvarum chromosomes, it would have been HO- (and separated from potential oppositemating-type spores by turbulence in the liquid medium), and could therefore have remained haploid. Whatever their origin, multiple temperature-selected clones exhibited chromosome rearrangements at 500 generations (Figure 6B). S. cerevisiae-like haploids likely acquired a competitive advantage at temperatures exceeding $35^{\circ} \mathrm{C}$, a speculation supported by reports that the cardinal growth temperature of $S$. cerevisiae is significantly greater than S. uvarum [35,36] and (Figure 3). Given that temperature tolerance is a multi-locus, quantitative trait [55], haploidization resulting in nearly-complete loss of the $S$. uvarum genome complement provides the shortest path to thermal tolerance for $S$. cerevisiae X $S$. uvarum hybrids. Further, haploid S. cerevisae are known to exhibit greater induced thermotolerance than isogenic diploids [56], and haploids outcompete diploids at elevated temperature $\left(37^{\circ} \mathrm{C}\right)$ when serially propagated in either YPD or low-nitrogen, minimal medium [57]. Consistent with these observations, after $48 \mathrm{~h}$ of growth at $40^{\circ} \mathrm{C}$ the haploid form of our S. cerevisiae parent attained higher cell density than the diploid $\left(\mathrm{A}_{600}=0.28 \pm 0.01 \mathrm{vs}\right.$. $0.19 \pm 0.01)$.

\section{Two species array comparative genomic hybridization} supports the inference that genome evolution in interspecific hybrid populations is selection-specific

To further characterize genomic changes that may have occurred during evolution at elevated temperatures or elevated ethanol, we performed array-Comparative Genomic Hybridization (a-CGH) on the temperature- and ethanolselected isolates as well as on parental strains and founding hybrids, using microarrays designed to uniquely detect hybridization to either the S. cerevisiae or the S. uvarum genomes, as described in [30]. Analysis of the parental strains indicates that our S. cerevisiae and S. uvarum isolates appear exactly as expected, i.e., they contain full genomes of their respective species with no chromosomal regions corresponding to the alternate species (Figure 8). The parental F1 hybrid contains, also as expected, a full set of chromosomes from both its $S$. cerevisiae and S. uvarum parents (Figure 8). Three isolates from the founder hybrid population were examined by a-CGH (See Additional file 1: Table S1). One (H1) appears to contain a 


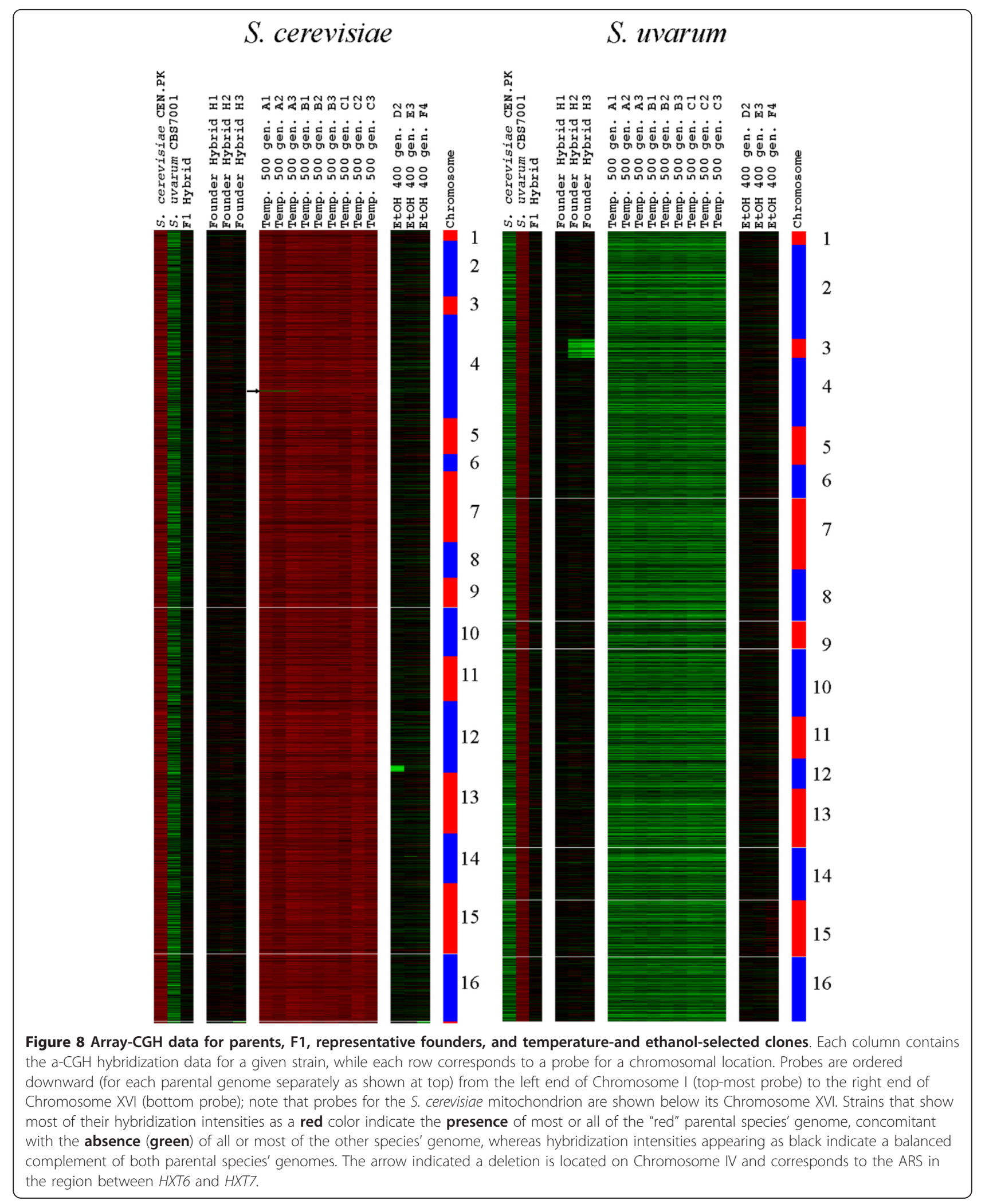


complete two-species genomic complement, and is thus possibly an F1 hybrid (Figure 8), consistent with its CHEF electrokaryotype (Figure 6A, lane 1). Two other isolates from the founder hybrid population ( $\mathrm{H} 2$ and $\mathrm{H} 3)$ lack $S$. uvarum Chromosome III (Figure 7), as was also seen by CHEF. At the level of resolution afforded by a-CGH we see no evidence for recombination between parental genomes in (Figure 7). Indeed, without a separate study on recombination in S. cerevisiae x $S$. uvarum hybrids it would be impossible to distinguish between an F1 that lost S. uvarum Chromosome III and an F2 that retained the majority of both parental genomes. Nevertheless, even given the likelihood of suppressed recombination between homeologous chromosomes, the founding hybrid population was genomically diverse, owing to random segregation of these homeologous chromosomes when the F1 hybrid underwent meiosis.

Using a-CGH we examined the same 9 temperatureselected isolates described above (A1 through A3, B1 through B3, and C1 through C3, Figure 1). For all 9 clones, the genome complement appears to be comprised solely of that of the S. cerevisiae parent (Figure 8). The a-CGH results for the three vessel A clones thus closely agree with their CHEF gel results (Figure 6B); furthermore, a-CGH reveals that all three vessel A clones contain a small deletion in the $S$. cerevisiae genome; because its length is only 1-3 kb it was undetectable on CHEF gels. This deletion is located on Chromosome IV and corresponds to the ARS in the region between HXT6 and HXT7 (Figure 8, indicated by an arrow). As the deletion appears identical among the three clones within the detection limits of a$\mathrm{CGH}$, it likely arose from the same member of the founder hybrid population within population A and does not represent multiple independent evolutionary events. Additional experiments will be required to ascertain both its prevalence in the vessel A population (i.e., whether it is "fixed"), as well as its possible adaptive value.

For the three 500-generation clones from population B and $\mathrm{C}$, our a-CGH results do not intuitively agree with the CHEF results. CHEF gel karyotypes for all B clones appear to show the presence of an additional chromosome; although the band is similar (but not identical) to mobility of S. uvarum Chromosome XI, a-CGH results from all assayed 500-generation vessel B clones show the presence of only the parental S. cerevisiae genome (Figure 8) indicating that the band must derive from a copy-neutral event within the $S$. cerevisiae genome. Similarly, the CHEF electrokaryotypes of the clones from population $\mathrm{C}$ show increase in size of at least two of the smaller chromosomes, but again, only the parental $S$. cerevisiae genome is present (Figure 8), suggesting the occurrence of copyneutral rearrangements.

We also performed a-CGH on 3 ethanol-selected isolates from each of Vessels D, E, and F (Figure 7). We investigated clones from the 400 generation time-point rather than at 500 generations, as we had observed greater karyotypic diversity in the 400 generation populations (Figure 6C vs. Additional file 5: Figure S4). An ethanol-selected clone from vessel D (Figure 7, EtOH 400 gen D2) appeared to be an essentially euploid F1 hybrid with a large deletion in the $S$. cerevisiae genome (but not deleted in the corresponding S. uvarum region): $100 \mathrm{~kb}$ of the right end of Chromosome XII, starting just proximal to (and including) the TUS1 gene and extending to the right telomere, is deleted in this isolate. Interestingly, Tus1 is involved in cell integrity signalling [58] and its deletion results in increased chitin deposition [59]; $E C M 30$, which is also included in the deleted region, is a gene possibly involved in cell wall biosynthesis. This deletion may explain the increased zymolyase resistance observed in Vessel D isolates (see Figure 5B). The Vessel $\mathrm{E}$ isolate examined by a- $\mathrm{CGH}$ again appeared to be an essentially euploid F1 (Figure 8, EtOH 400 gen E3), but exhibited a $15 \mathrm{~kb}$ deletion on its $S$. cerevisiae Chromosome XIV starting between MEP2 and $A A H 1$ and extending to a tRNA gene just beyond FYV6. The deleted region contains genes involved in a variety of functions including nitrogen utilization $(A A H 1)$, recombination (THO2 and FYV6) and chromatin modifications (EAF7 and FPR1). The ethanol-selected isolate from Vessel $\mathrm{F}$ lost its $S$. cerevisiae mitochondrial genome, yet otherwise appeared as an intact euploid F1 hybrid (Figure 8, EtOH 400 gen F4).

\section{Conclusions}

This study is the first to report how populations of interspecific hybrid organisms evolving in the laboratory follow dramatically different evolutionary trajectories depending on the selective pressures applied. Indeed, to the best of our knowledge this is the first long-term study in experimental microbial evolution initiated with a heterogenous population of genetically diverse variants rather than with a homogenous population derived from a single clone. This collection of variants was used to found six replicate populations that evolved under glucose-sufficient, nitrogen-limiting conditions: three under temperature selection and three under ethanol selection.

In clones that arose under temperature selection, thermal tolerance was significantly greater than that of isolates in the founder population and of the parental F1 strain, a result likely due in part to segregational loss of the temperature-sensitive $S$. uvarum genome, resulting in S. cerevisiae haploids. This event occurred independently in all populations under temperature selection, although the haploid variants arose at different times and swept their respective populations over different time intervals. Interestingly, loss of heterozygosity under thermal and oxidative stress has recently been documented in 
Candida albicans, where increased stress appears to elevate rates of recombination, including chromosome missegration [42]. In diploid $S$. cerevisiae, haploidization has now been shown to provide an escape for persistent DNA rearrangement stress due to the presence of mutator alleles [60]. Thus, strong selection to augment thermal tolerance as well as to diminish DNA stress may help to explain the outcome of temperature selection on a population of newly-formed interspecific hybrids.

Heritable thermotolerance among these strains was evident in their ability to grow at temperatures restrictive to founding hybrid isolates on solid (data not shown) and in liquid media, as well as in their intrinsic and acquired resistance to heat shock. Temperature-selected strains were also ethanol resistant, most likely due to loss of the ethanol-sensitive S. uvarum genome [38]. Based on CHEF analysis, two of three temperature-selected populations came to be dominated by a unique karyotype, while one remained polymorphic, indicating multiple pathways to the evolution of thermotolerance. Evolution of multiple heat stress tolerance mechanisms was also manifest as differential, strain-specific resistance to zymolyase digestion as well as to Micafungin, a drug that targets cell wall biosynthesis. Because increased zymolyase resistance correlates with increased ethanol resistance $[61,62]$, adaptive changes in cell wall integrity in temperature-selected clones may underlie "cross-protection" against ethanol stress.

Under temperature selection, adaptive changes in zymolyase and Micafungin resistance could be expected inasmuch as cell wall integrity has been shown to play a key role in thermal stress response [63]. Acquisition of thermotolerance in diauxic and stationary phase yeast has also been linked to accumulation of the disaccharide trehalose $[64,65]$, however, we uncovered no evidence that trehalose hyper-accumulates in heat-shock resistant clones that arose under temperature selection (data not shown).

We uncovered little evidence for adaptive evolution of ethanol tolerance. Populations evolved under ethanol selection became dominated by euploid $S$. cerevisiae $\mathrm{x}$ S. uvarum hybrids. These hybrids showed improved growth on ethanol-amended media relative to some (e.g., founder H3), but not all founding hybrids, nor to the parental F1 strain. Evidence suggesting heritable changes in cell wall composition in these isolates was limited to higher (but not significantly higher) zymolyase resistance. We therefore conclude that a subset of euploid hybrids in the original founder population was at or near a fitness peak for ethanol tolerance [38], providing limited scope for selection.

It is important to bear in mind that temperature and ethanol tolerance assays were performed on only a few clones that together represent a small fraction of the genetic variation latent in our terminal populations.
Other clones may exist that exhibit even greater thermal tolerance and, perhaps also greater ethanol tolerance. Indeed, in follow-up experiments we found that the temperature-selected clones we had randomly chosen for analysis grew poorly in chemostat monoculture at $>45^{\circ} \mathrm{C}$, suggesting that other more highly tolerant variants exist in the terminal populations. Also, it may be that a slow ramp-up in temperature may be required even for thermotolerant clones to achieve their true performance maxima. We acknowledge the possibility that highly stress tolerant variants may have been present in the hybrid founder population used for these experiments. Such clones may have persisted at low frequency under slow growth conditions $\left(\mathrm{D}=0.15 \mathrm{~h}^{-1}\right)$ until selection favored them over more abundant, stress-sensitive clones. This possibility highlights an outstanding unresolved issue in experimental evolution, namely the extent to which adaptation results from accumulation of $d e$ novo mutations as opposed to selection of rare adaptive mutants that may exist at the onset of selection. In this regard, it would be interesting to perform a highthroughput phenotypic screen of hundreds of variants in the founder and terminal populations, as well as to perform population sequencing at the beginning and end of these experiments. Still, given that selection was applied over the course of 500 generations it is virtually certain that multiple de novo mutations distinguish members of the terminal populations from their common ancestors.

The industrial applications of hybrid variability have already been recognized [66], and our approach of selecting on a diverse hybrid population may be used to enhance routine industrial strain development. However, our findings highlight the need to carefully choose appropriate parental strains as the selection process cannot rely solely on hybrid vigor. Genome plasticity under strong selection may lead to unexpected results, such as the shedding of one or another parental genome. In our experiments this remarkable occurrence seems to provide the most direct route to thermal tolerance, a trait whose many genetic determinants are widely distributed across the S. uvarum and S. cerevisiae genomes. Selection on a genetically diverse population of $S$. cerevisiae alone might produce comparable gains in fitness at high temperatures. Indeed, wild isolates of that species have been isolated which can grow at temperatures exceeding $45^{\circ} \mathrm{C}$ [36], and genome shuffling experiments [67] involving recursive protoplast fusions have produced $S$. cerevisiae strains that aggressively ferment at temperatures up to $48^{\circ} \mathrm{C}$ [68]. Most importantly, our findings highlight the importance of the ecological theatre in determining the outcome of the evolutionary play. Euploid interspecific $S$. cerevisiae x $S$. uvarum hybrids are genetically stable and highly fit as ambient levels of ethanol increase, but poorly fit under rising temperature. Thus, 
the evolutionary fate of hybrids in nature likely depends as much on their environmental context as on their genetic potential.

\section{Methods}

\section{Strains and hybrid creation}

All yeast strains used in this work are derivatives of the prototrophic diploid strains CEN.PK (Saccharomyces cerevisiae) [44] and CBS7001 (S. uvarum) [30] the former obtained from D. Botstein, the latter from E. Louis. To obtain F1 hybrids, S. cerevisiae CEN.PK and $S$. uvarum CBS7001 were transformed to G418 and hygromycin antibiotic resistance, respectively, using $2 \mu$-based YEp352-KanMX and YEp352-hph plasmids. After verifying plasmid segregation, transformants were sporulated for three days on sporulation medium (1\% potassium acetate, $0.1 \%$ yeast extract and $0.05 \%$ glucose) and then mixed and plated on rich medium supplied with G418 and hygromycin at $200 \mu \mathrm{g} \mathrm{mL} \mathrm{m}^{-1}$ and $300 \mu \mathrm{g} \mathrm{mL}{ }^{-1}$, respectively. F1 progeny were selected as clones resistant to both antibiotics. After confirming segregational loss of both plasmids, a single F1 clone was sporulated for 3 days in liquid sporulation medium (1\% potassium acetate); unsporulated cells were then digested by a combination of Zymolyase T100 and a detergent, as described in [33], leaving F2 hybrid spores, which represent rare viable spore progeny of the F1 clone. These spores were left to germinate and mate overnight, then after verifying cell titer, spread on 48 large plates so that every cell could grow into a colony, unencumbered by others. Approximately 10,000 colonies were washed with $5 \mathrm{~mL}$ of sterile $\mathrm{dd}_{2} \mathrm{O}$ per plate and combined to make the initial hybrid pool.

\section{Media and growth conditions}

Unless otherwise indicated, all media used was the inorganic nitrogen-limiting $\left(0.15 \mathrm{~g} \mathrm{~L}^{-1}\left(\mathrm{NH}_{4}\right)_{2} \mathrm{SO}_{4}\right)$ medium used for batch and chemostat cultures described by Verduyn et al. [69]. For chemostat experiments $10 \mathrm{~L}$ of basal medium were prepared in $13 \mathrm{~L}$ glass carboys. To each liter a post-sterile addition was made of: $1.0 \mathrm{~mL}$ $1000 \times$ vitamins, $1.0 \mathrm{~mL} 1000 \times$ trace metals, and 45.0 $\mathrm{mL} 20 \%$ glucose (final conc. $9 \mathrm{~g} \mathrm{~L}^{-1}$ ) [69], a formulation hereafter referred to as low-nitrogen, minimal medium. Populations were cultured in water-jacketed chemostats (200 mL working volume), which were mixed and aerated using sterile, humidified house air at a flow rate of $10 \mathrm{~L} \mathrm{~h}^{-1}$ (0.8 vvm). For temperature selections, triplicate experimental populations were founded by adding cells from an inoculum prepared as described to a final density of $\sim 10^{8}$ cells per $\mathrm{mL}$, with the initial target dilution rate set at $\mathrm{D}=0.15 \mathrm{~h}^{-1}$ and the initial temperature at $31^{\circ} \mathrm{C}$. Every 25 generations (about every week), culture temperature was increased by $1^{\circ} \mathrm{C}$; as cell yield declined steeply above $37^{\circ} \mathrm{C}$, later adjustments were made on a bi-weekly basis. To avoid wash-out, dilution rate at higher temperatures was lowered to $\mathrm{D}=0.05 \mathrm{~h}^{-1}$ at $41^{\circ}$ $\mathrm{C}$ and remained at this level until 500 generations. The final vessel temperature at 500 generations was $46.5^{\circ} \mathrm{C}$. For ethanol selections, the same founding population was used to inoculate three identical $200 \mathrm{~mL}$ chemostat vessels, which were kept at room temperature $\left(25-28^{\circ} \mathrm{C}\right)$. The basal medium was identical to that used for temperature selection; ethanol content of this medium was increased by $1 \%$ approximately every $10 \mathrm{~d}$. Evaporation was impeded by layering sterile mineral oil atop ethanol-amended, low-nitrogen minimal medium. Because cell growth was strongly inhibited at ethanol amendments $>12 \%$, at these concentrations chemostat dilution rate was reduced to $\mathrm{D}=0.05 \mathrm{~h}^{-1}$ to prevent wash-out.

\section{Sampling and assay of growth parameters}

Optical density at $\lambda=600 \mathrm{~nm}$ was measured daily using a Biomate3 spectrophotometer (Thermo Electron Corp, Waltham, MA, USA.) by sterile removal of $1 \mathrm{~mL}$ of culture from each vessel, and measuring absorbance of 1:10 dilutions. Approximately every 15 generations $5 \mathrm{~mL}$ were removed from each vessel (i) to archive samples as $15 \%$ glycerol stocks at $-80^{\circ} \mathrm{C}$, (ii) to estimate viable cell counts by plating serial dilutions on YPD, and (iii) to determine concentrations of glucose and ethanol, as described below. Three-mL aliquots archived for analysis of residual growth substrate and ethanol were filtered using a $0.2 \mu \mathrm{m}$ nylon filter and stored at $-20^{\circ} \mathrm{C}$ until assayed.

\section{Temperature and ethanol tolerance assays Isolates tested}

For follow-up experiments we used the diploid parental strains S. cerevisiae CEN.PK and S. uvarum CBS7001, the F1 hybrid, three isolates from the founder hybrid population, and isolates from each of six experimental populations at the final 500-generation time-point. An overview of how the hybrid population was generated and the naming scheme for the isolates is shown in Figure 1 , and a detailed list of all tested evolved isolates is presented in Additional file 1: Table S1. Representatives of the founder hybrid population are the same isolates shown in Figure 6A, lanes 1, 2, 3, and are referred to as $\mathrm{H} 1, \mathrm{H} 2$, and $\mathrm{H} 3$. Temperature selected isolates tested are shown in Figure 6B Lanes 1, 2, 3 (Temperature selected A1, A2, A3); Lanes 8, 9, 10 (Temperature selected B1, B2, B3); and Lanes 15, 16, 17 (Temperature selected C1, C2, C3). Ethanol evolved isolates tested are shown in Figure 6C Lanes 1, 2, 3 (Ethanol selected D1, D2, D3); Lanes 9, 10, 11 (Ethanol selected E1, E2, E3); and Lanes 16, 17, 18 (Ethanol selected F1, F2, F3). In certain instances, to test the performance of the ancestral haploid form we included a $S$. cerevisiae CEN.PK 
haploid. For each experimental parameter tested, individual isolates were run in triplicate.

\section{Growth in liquid media}

For all experiments we used parental isolates, F1, 3 founding hybrid isolates, and three isolates from each terminal population (Figure 1). Pre-cultures were grown overnight in 200 Lof low-nitrogen, minimal medium at $25^{\circ} \mathrm{C}$ and used to inoculate test cultures to a starting $\mathrm{A}_{600}$ of 0.01 . All test cultures were similarly grown in $200 \mu \mathrm{L}$ of lownitrogen, minimal medium in 96 -well microtiter plates. To assay temperature tolerance, cultures were grown at $40^{\circ} \mathrm{C}$; to test ethanol tolerance cultures were grown at $25^{\circ} \mathrm{C}$ in the same medium, amended with $8 \%$ ethanol. All cultures were grown in triplicate to stationary phase $(48 \mathrm{~h})$. Optical density was measured spectrophotometrically at $\lambda=600$ $\mathrm{nm}$ every $6 \mathrm{~h}$ to confirm that all cultures were in stationary phase before the final measure (Spectramax 340PC, Molecular Devices, Sunnyvale, CA, USA). Replicate estimates of growth parameters for temperature or ethanol isolates (A1-F3) were pooled by vessel for statistical comparison to the parent, F1 and founding strains.

\section{Induced versus inherent thermotolerance}

Each parental stain, the F1, the three representative founder stains and two isolates from each temperature selection (isolates 1 and 2 from each vessel in Additional file 1: Table S1) were grown in triplicate overnight at $25^{\circ} \mathrm{C}$ in $50 \mathrm{~mL}$ low-nitrogen, minimal medium to $\mathrm{A}_{600}$ 0.4-0.6. To test strain-specific differences in induced versus inherent thermotolerance, each culture was apportioned into two vessels: one was placed in a $37^{\circ} \mathrm{C}$ water bath for $5 \mathrm{~min}$ and then incubated at $37^{\circ} \mathrm{C}$ on a shaker (induced thermotolerance), while the other was shaken at room temperature (inherent thermotolerance). After $50 \mathrm{~min}$, cultures were diluted into fresh pre-warmed media, then placed in a $48^{\circ} \mathrm{C}$ water bath, whereafter samples were removed every hour for $5 \mathrm{~h}$ and diluted before plating onto YPD agar. Survivorship was reported as the percentage of viable cells remaining at each timepoint, relative to viable cell counts at $\mathrm{T}=0$ hours.

\section{Analysis of cell wall phenotypes}

To determine whether observed changes in thermal tolerance were correlated with changes in cell wall composition we performed a spheroplast assay, as described in [53]. Cultures were diluted to an $\mathrm{A}_{600}=0.8$ in spheroplast medium (1.2 M sorbitol), whereupon zymolyase was added to achieve a final assay concentration of 250 $\mu \mathrm{g} \mathrm{mL}{ }^{-1}$. Decrease in $\mathrm{A}_{600}$, resulting from cell wall digestion, was measured over the course of $1 \mathrm{~h}$ at $37^{\circ} \mathrm{C}$. All strains were tested in triplicate, and the selected isolates used were the "1" isolates (e.g., A1, B1, etc.) from each evolved population (Additional file 1: Table S1).
Strain-specific resistance to Micafungin (Astellas Pharma, Tokyo), a compound that targets fungal cell wall biosynthesis was tested by culturing cells at $25^{\circ} \mathrm{C}$ in $200 \mu \mathrm{L}$ of low-nitrogen, with $150 \mathrm{nM}$ Micafungin or a solvent control (DMSO). Cells were inoculated to an $\mathrm{A}_{600}=0.01$ and incubated until all cultures were in stationary phase and measured spectrophotometrically. Growth was calculated relative to the solvent-only control. All isolates were run in triplicate.

\section{Genomic analyses}

CHEF analysis was conducted as previously described $[70,71]$. To assay ploidy flow cytometry was performed using SYTOX green as described in [72]. Microarraybased Comparative Genome Hybridization (array-CGH) was performed as described in [30]. Microarray data have been deposited in the GEO repository under accession GSE24479.

\section{Statistics}

We used one-way ANOVA to compare differences in response to temperature and ethanol tolerance, zymolyase, and Micafungin resistance, using Tukey's HSD. For individual comparisons we used a T-test. We used Sigma Plot 11 (Dundas software LTD, Germany) for all statistical analyses.

\section{Additional material}

Additional file 1: Table S1 Founding hybrid and selected isolates used in genetic and physiological experiments. Reference to each strain's CHEF karyotype is presented is column 2.

Additional file 2: Figure S1 Growth of evolved isolates with $8 \%$ ethanol supplementation. Culture density (A600) of parental, F1,

founding and selected hybrid strains from each experimental population following $48 \mathrm{~h}$ growth in liquid, low-nitrogen, minimal medium at $25^{\circ} \mathrm{C}$ in medium, amended with $8 \%$ ethanol.

Additional file 3: Figure S2 Assay of Micafungin sensitivity of parental species, founding hybrids, temperature-selected hybrids, and ethanol selected hybrids. Growth of parental and temperatureselected hybrids in low-nitrogen, minimal medium at $25^{\circ} \mathrm{C}$, supplemented with $150 \mathrm{nM}$ of Micafungin. Presented is the growth $\left(A_{600}\right)$ relative to the solvent (DMSO) control of the 3 replicate cultures in stationary phase. Asterisks indicate that isolates in vessels $A$ and $C$ had significantly greater Micafungin resistance than all other isolates $(P<$ 0.05 , Mean \pm S.E.).

Additional file 4: Figure S3 Changes in ploidy within experimentally selected populations. Cell populations were stained with SYTOX Green and sorted by flow cytometry as described. (A) S. cerevisiae haploid and S. cerevisiae/S. uvarum diploid. (B) Vessel A: 101gen (2 N), 157gen (mixed $2 \mathrm{~N}+1 \mathrm{~N}$ ), 224gen (1 N); (C) Vessel B: 115gen (2 N), 125gen (mixed $2 \mathrm{~N}$ $+1 \mathrm{~N}$ ), 135gen (1 N); and (D) Vessel C: 43gen (2 N), 51gen (mixed $2 \mathrm{~N}+$ $1 \mathrm{~N})$, 95gen (1 N).

Additional file 5: Figure S4 CHEF karyotypes in three experimental populations after $\mathbf{4 0 0}$ generations of nitrogen-limited, glucosesufficient culture with increasing ethanol. At left are the karyotypes of parental strains, S. cerevisiae CEN.PK, S. uvarum CBS7001, and their F1 interspecific hybrid. 7 random clones were isolated from each experimental population. Green arrows indicate karyotypic variability in experimental populations. Medium ethanol content at 400 generations was $14 \%$. 


\section{Acknowledgements}

The authors wish to thank Carla Boulianne-Larsen and Jeff Good for critically reading the manuscript. Gregory Koniges performed heat shock experiments to distinguish between induced and inherent thermal resistance. Flow cytometry was performed with the assistance of Pamela Shaw. We thank Minoru Yoshida and Astellas Pharma for the use of Micafungin. This work was supported by grants to GS from Global Climate and Energy Project (Grant \#33450) and to FR from NASA NNX07AJ28G, as well as by NIH grant P20RR017670 to the University of Montana Fluorescence Cytometry Core Facility.

\section{Author details}

${ }^{1}$ Chemical Genomics Research Group, RIKEN Advance Science Institute, Wako, Wako, Japan. ${ }^{2}$ School of Chemical and Biotechnology, SASTRA University, Tirumalaisamudram Thanjavur- 613401, Tamil Nadu, India. ${ }^{3}$ Division of Biological Sciences, The University of Montana, Missoula MT 59812, USA. ${ }^{4}$ DuPont Corporation, Wilmington DE 19880, USA. ${ }^{5}$ Department of Genetics, Stanford University School of Medicine, Stanford, CA 943055120, USA.

\section{Authors' contributions}

JP, EK, SN, AK, and RFR designed and conducted all selection and physiological experiments. EK created the hybrid strains. KC, AS and EK conducted CHEF analysis. BD and GS designed and performed a-CGH analyses. All authors read and approved the final manuscript.

Received: 13 November 2011 Accepted: 2 April 2012 Published: 2 April 2012

\section{References}

1. Gompert Z, Fordyce JA, Forister ML, Shapiro AM, Nice CC: Homoploid hybrid speciation in an extreme habitat. Science 2006, 314:1923-1925.

2. Mallet J: Hybrid speciation. Nature 2007, 446:279-283.

3. Mercer KL, Andow DA, Wyse DL, Shaw RG: Stress and domestication traits increase the relative fitness of crop-wild hybrids in sunflower. Ecol Lett 2007, 10:383-393.

4. Sexton JP, McIntyre PJ, Angert AL, Rice KJ: Evolution and ecology of species range limits. Ann Rev Ecol Evol Systemat 2009, 40:415-436.

5. Gross BL, Rieseberg LH: The ecological genetics of homoploid hybrid speciation. J Hered 2005, 96:241-252.

6. Meyer A, Salzburger W, Schartl M: Hybrid origin of a swordtail species (Teleostei: Xiphophorus clemenciae) driven by sexual selection. Mol Ecol 2006, 15:721-730.

7. Rieseberg LH, Raymond O, Rosenthal DM, Lai Z, Livingstone K, Nakazato T, Durphy JL, Schwarzbach AE, Donovan LA, Lexer C: Major ecological transitions in wild sunflowers facilitated by hybridization. Science 2003, 301:1211-1216.

8. Nolte AW, Tautz D: Understanding the onset of hybrid speciation. Trends Genet 2010, 26:54-58.

9. East EM: Heterosis. Genetics 1936, 21:375-397.

10. Darwin C: In The effects of cross and self fertilisation in the vegetable kingdom Edited by: Murray J 1900.

11. Coyne JA, Orr HA: Patterns of speciation in Drosophila. Evolution 1989, 43:362-381.

12. Alipaz JA, Fang S, Osada N, Wu C: Evolution of sexual isolation during secondary contact: genotypic versus phenotypic changes in laboratory populations. Am Nat 2005, 165:420-428.

13. Alipaz JA, Karr TL, Wu C-I: Evolution of sexual isolation in laboratory populations: fitness differences between mating types and the associated hybrid incompatibilities. Am Nat 2005, 165:429-438.

14. Lenski RE, Rose MR, Simpson SC, Tadler SC: Long-term experimental evolution in Escherichia coli. I. adaptation and divergence during 2,000 generations. Am Nat 1991, 138:1315-1341

15. Travisano M, Mongold J, Bennett A, Lenski R: Experimental tests of the roles of adaptation, chance, and history in evolution. Science 1995, 267:87-90.

16. Paquin $C E$, Adams J: Relative fitness can decrease in evolving asexual populations ofS. cerevisiae. Nature 1983, 306:368-371.

17. Stelkens R, Seehausen O: Genetic distance between species predicts novel trait expression in their hybrids. Evolution 2009, 63:884-897.
18. Kvitek DJ, Will JL, Gasch AP: Variations in stress sensitivity and genomic expression in diverse S. cerevisiae isolates. PLoS Genet 2008, 4:e1000223.

19. Ferea TL, Botstein D, Brown PO, Rosenzweig RF: Systematic changes in gene expression patterns following adaptive evolution in yeast. Proc Nat Acad Sci USA 1999, 96:9721-9726.

20. Zeyl C: Capturing the adaptive mutation in yeast. Res Microbiol 2004, 155:217-223.

21. Liti G, Louis EJ: Yeast evolution and comparative genomics. Ann Rev Microbiol 2005, 59:135-153.

22. Wenger JW, Piotrowski J, Nagarajan S, Chiotti K, Sherlock G, Rosenzweig F: Hunger artists: yeast adapted to carbon limitation show trade-offs under carbon sufficiency. PLoS Genet 2011, 7:e1002202.

23. Dunham MJ, Badrane H, Ferea T, Adams J, Brown PO, Rosenzweig F, Botstein D: Characteristic genome rearrangements in experimental evolution of Saccharomyces cerevisiae. Proc Nat Acad Sci USA 2002, 99:16144-16149.

24. Gresham D, Desai MM, Tucker CM, Jenq HT, Pai DA, Ward A, DeSevo CG, Botstein D, Dunham MJ, Snyder M: The repertoire and dynamics of evolutionary adaptations to controlled nutrient-limited environments in yeast. PLoS Genet 2008, 4:e1000303.

25. Greig D, Travisano M, Louis EJ, Borts RH: A role for the mismatch repair system during incipient speciation in Saccharomyces. J Evol Biol 2003, 16:429-437.

26. Libkind D, Hittinger $C T$, Valério E, Gonçalves C, Dover J, Johnston $M$, Gonçalves P, Sampaio JP: Microbe domestication and the identification of the wild genetic stock of lager-brewing yeast. Proc Nat Acad Sci USA 2011, 108:14539-14544.

27. Kuehne HA, Murphy HA, Francis CA, Sniegowski PD: Allopatric divergence, secondary contact, and genetic isolation in wild yeast populations. Curr Biol 2007, 17:407-411.

28. Dujon B, Sherman D, Fischer G, Durrens P, Casaregola S, Lafontaine I, de Montigny J, Marck C, Neuveglise C, Talla E, Goffard N, Frangeul L, Aigle M, Anthouard V, Babour A, Barbe V, Barnay S, Blanchin S, Beckerich J-M, Beyne E, Bleykasten C, Boisrame A, Boyer J, Cattolico L, Confanioleri F, de Daruvar A, Despons L, Fabre E, Fairhead C, Ferry-Dumazet H, Groppi A, Hantraye $F$, Hennequin C, Jauniaux N, Joyet $P$, Kachouri R, Kerrest A, Koszul R, Lemaire M, Lesur I, Ma L, Muller H, Nicaud J-M, Nikolski M, Oztas S, Ozier-Kalogeropoulos O, Pellenz S, Potier S, Richard G-F, Straub M-L, Suleau A, Swennen D, Tekaia F, Wesolowski-Louvel M, Westhof E, Wirth B, Zeniou-Meyer M, Zivanovic I, Bolotin-Fukuhara M, Thierry A, Bouchier C, Caudron B, Scarpelli C, Gaillardin C, Weissenbach J, Wincker P, Souciet J-L: Genome evolution in yeasts. Nature 2004, 430:35-44.

29. Wolfe KH: Comparative genomics and genome evolution in yeasts. Philos Trans Roy Soc B 2006, 361:403-412.

30. Dunn B, Sherlock G: Reconstruction of the genome origins and evolution of the hybrid lager yeast Saccharomyces pastorianus. Genome Res 2008, 18:1610-1623.

31. Bulter T, Alcalde M, Sieber V, Meinhold P, Schlachtbauer C, Arnold FH: Functional expression of a fungal laccase in Saccharomyces cerevisiae by directed evolution. Appl Environ Microbiol 2003, 69:987-995.

32. Edgardo A, Carolina P, Manuel R, Juanita F, Baeza J: Selection of thermotolerant yeast strains Saccharomyces cerevisiae for bioethanol production. Enzy Microb Technol 2008, 43:120-123.

33. Greig D, Louis EJ, Borts RH, Travisano M: Hybrid speciation in experimental populations of yeast. Science 2002, 298:1773-1775.

34. Kellis M, Patterson N, Endrizzi M, Birren B, Lander ES: Sequencing and comparison of yeast species to identify genes and regulatory elements. Nature 2003, 423:241-254.

35. Rainieri S, Zambonelli C, Giudici P, Castellari L: Characterisation of thermotolerant Saccharomyces cerevisiae hybrids. Biotechnol Lett 1998, 20:543-547.

36. Salvadó Z, Arroyo-López FN, Guillamón JM, Salazar G, Querol A, Barrio E: Temperature adaptation markedly determines evolution within the genus accharomyces. Appl Environ Microbiol 2011, 77:2292-2302.

37. Piper PW: The heat shock and ethanol stress responses of yeast exhibit extensive similarity and functional overlap. FEMS Microbiol Lett 1995, 134:121-127.

38. Arroyo-López FN, Salvadó Z, Tronchoni J, Guillamón JM, Barrio E, Querol A: Susceptibility and resistance to ethanol in Saccharomyces strains isolated from wild and fermentative environments. Yeast 2010, 27:1005-1015. 
39. Belloch C, Orlic S, Barrio E, Querol A: Fermentative stress adaptation of hybrids within the Saccharomyces sensu stricto complex. Int/ J Food Microbiol 2008, 122:188-195.

40. Vitousek P, Howarth R: Nitrogen limitation on land and in the sea: how can it occur? Biogeochem 1991, 13:87-115.

41. Goddard MR, Bradford MA: The adaptive response of a natural microbia population to carbon- and nitrogen-limitation. Ecol Lett 2003, 6:594-598.

42. Forche A, Abbey D, Pisithkul T, Weinzierl MA, Ringstrom T, Bruck D, Petersen K, Berman J: Stress alters rates and types of loss of heterozygosity in Candida albicans. mBio 2:e00129-11.

43. Trollmo C, André L, Blomberg A, Adler L: Physiological overlap between osmotolerance and thermotolerance in Saccharomyces cerevisiae. FEMS Microbiol Lett 1988, 56:321-325.

44. van Dijken JP, Bauer J, Brambilla L, Duboc P, Francois JM, Gancedo C, Giuseppin MLF, Heijnen JJ, Hoare M, Lange HC, Madden EA, Niederberger P, Nielsen J, Parrou $J$, Petit T, Porro D, Reuss M, van Riel N, Rizzi M, Steensma HY, Verrips CT, Vindeløv J, Pronk JT: An interlaboratory comparison of physiological and genetic properties of four Saccharomyces cerevisiae strains. Enzy Microb Technol 2000, 26:706-714.

45. Godfrey MH: Hybrid zones-natural laboratories for evolutionary studies. Trends Ecol Evol 1988, 3:158-167.

46. Schulze U, Lidén G, Nielsen J, Villadsen J: Physiological effects of nitrogen starvation in an anaerobic batch culture of Saccharomyces cerevisiae. Microbiology 1996, 142:2299-2310.

47. Thomsson E, Gustafsson L, Larsson C: Starvation response of Saccharomyces cerevisiae grown in anaerobic nitrogen- or carbon-limited chemostat cultures. Appl Environ Microbiol 2005, 71:3007-3013.

48. Allen C, Büttner S, Aragon AD, Thomas JA, Meirelles O, Jaetao JE, Benn D, Ruby SW, Veenhuis M, Madeo F, Werner-Washburne M: Isolation of quiescent and nonquiescent cells from yeast stationary-phase cultures. $J$ Cell Biol 2006, 174:89-100.

49. Piper P: Induction of heat shock proteins and thermotolerance. In In Yeast Protocols. Volume 53. New Jersey: Humana Press; 1996:313-318.

50. Piper PW: Molecular events associated with acquisition of heat tolerance by the yeast Saccharomyces cerevisiae. FEMS Microbiol Rev 1993, 11:339-355.

51. Aguilar-Uscanga B, François JM: A study of the yeast cell wall composition and structure in response to growth conditions and mode of cultivation. Lett Appl Microbiol 2003, 37:268-274.

52. Lesage G, Bussey H: Cell wall assembly in Saccharomyces cerevisiae. Microbiol Mol Biol Rev 2006, 70:317-343.

53. Ovalle R, Lim ST, Holder B, Jue CK, Moore CW, Lipke PN: A spheroplast rate assay for determination of cell wall integrity in yeast. Yeast 1998, 14:1159-1166.

54. Denning DW: Echinocandins: a new class of antifungal. J Antimicrob Chemother 2002, 49:889-891.

55. Steinmetz LM, Sinha H, Richards DR, Spiegelman Jl, Oefner PJ, McCusker JH, Davis RW: Dissecting the architecture of a quantitative trait locus in yeast. Nature 2002, 416:326-330.

56. Piper PW, Davies MW, Curran B, Lockheart A, Spalding A, Tuite MF: The influence of cell ploidy on the thermotolerance of Saccharomyces cerevisiae. Curr Genet 1987, 11:595-598.

57. Mable BK: Ploidy evolution in the yeast Saccharomyces cerevisiae: a test of the nutrient limitation hypothesis. J Evol Biol 2001, 14:157-170.

58. Schmelzle T, Helliwell SB, Hall MN: Yeast protein kinases and the RHO1 exchange factor TUS1 are novel components of the cell integrity pathway in yeast. Mol Cell Biol 2002, 22:1329-1339.

59. Lesage G, Shapiro J, Specht CA, Sdicu A-M, Menard P, Hussein S, Tong AHY, Boone $\mathrm{C}$, Bussey $\mathrm{H}$ : An interactional network of genes involved in chitin synthesis in Saccharomyces cerevisiae. BMC Genet 2005, 6:8.

60. Alabrudzinska M, Skoneczny M, Skoneczna A: Dipoid-specific genome stability genes of $S$. cerevisiae: genomic screen reveals haploidization as an escape from persisting DNA rearrangement stress. PLoS One 2011, 6: e21124.

61. Yazawa $H$, Iwahashi $H$, Uemura $H$ : Disruption of URA7 and GAL6 improves the ethanol tolerance and fermentation capacity of Saccharomyces cerevisiae. Yeast 2007, 24:551-560.

62. Anderson MJ, Barker SL, Boone C, Measday V: Identification of RCN1 and RSA3 as ethanoltolerant genes in Saccharomyces cerevisiae using a high copy barcoded library. FEMS Yeast Res 2012, 12:48-60.
63. Verna J, Lodder A, Lee K, Vagts A, Ballester R: A family of genes required for maintenance of cell wall integrity and for the stress response in Saccharomyces cerevisiae. Proc Nat Acad Sci USA 1997, 94:13804-13809.

64. Li L, Ye $Y$, Pan $L$, Zhu $Y$, Zheng $S$, Lin $Y$ : The induction of trehalose and glycerol in Saccharomyces cerevisiae in response to various stresses. Biochem Biophys Res Comm 2009, 387:778-783

65. Van Dijck P, Colavizza D, Smet P, Thevelein J: Differential importance of trehalose in stress resistance in fermenting and nonfermenting Saccharomyces cerevisiae cells. Appl Environ Microbiol 1995, 61:109-115.

66. Jimenez J, Benitez T: Selection of ethanol-tolerant yeast hybrids in $\mathrm{pH}$ regulated continuous culture. Appl Environ Microbiol 1988, 54:917-922.

67. Stephanopoulos G: Metabolic engineering by genome shuffling. Nat Biotech 2002, 20:666-668.

68. D-jian S, C-lu W, K-ming W: Genome shuffling to improve thermotolerance, ethanol tolerance and ethanol productivity of Saccharomyces cerevisiae. J Ind Microbiol Biotechnol 2008, 36:139-147.

69. Verduyn C, Postma E, Scheffers WA, Van Dijken JP: Effect of benzoic acid on metabolic fluxes in yeasts: a continuous-culture study on the regulation of respiration and alcoholic fermentation. Yeast 1992, 8:501-517.

70. Chu G, Vollrath D, Davis RW: Separation of large DNA molecules by contour-clamped homogeneous electric fields. Science 1986, 234:1582-1585.

71. Carle GF, Olson MV: An electrophoretic karyotype for yeast. Proc Nat Acad Sci USA 1985, 82:3756-3760

72. Haase SB, Reed SI: Improved flow cytometric analysis of the budding yeast cell cycle. Cell Cycle 2002, 1:132-136.

doi:10.1186/1471-2148-12-46

Cite this article as: Piotrowski et al:: Different selective pressures lead to different genomic outcomes as newly-formed hybrid yeasts evolve. BMC Evolutionary Biology 2012 12:46.

\section{Submit your next manuscript to BioMed Central and take full advantage of:}

- Convenient online submission

- Thorough peer review

- No space constraints or color figure charges

- Immediate publication on acceptance

- Inclusion in PubMed, CAS, Scopus and Google Scholar

- Research which is freely available for redistribution

Submit your manuscript at www.biomedcentral.com/submit
C Biomed Central 